\title{
Interfacial instability in a time-periodic rotating Hele-Shaw Cell
}

\author{
J. Bouchgl ${ }^{1}$, S. Aniss ${ }^{1, a}$, M. Souhar ${ }^{2}$, and A. Hifdi ${ }^{1}$ \\ 1 University Hassan II Aïn-Chock, Faculty of Sciences, Laboratory of Mechanics, Casablanca, Morocco \\ 2 Lemta UMR CNRS 7563 Ensem, 2 avenue de la Forêt de Haye, BP 160, Vandoeuvre Lès-Nancy 54504, France
}

\begin{abstract}
The effect of time-periodic angular velocity on the interfacial instability of two immiscible, viscous fluids of different densities and confined in an annular Hele-Shaw cell is investigated. An inviscid linear stability analysis of the viscous and time dependent basic flow leads to a periodic Mathieu oscillator describing the evolution of the interfacial amplitude. We show that the relevant parameters that control the interface are the Bond number, viscosity ratio, Atwood number and the frequency number.
\end{abstract}

\section{Introduction}

Several theoretical and experimental works have been carried out to study the interfacial instability in an annular Hele-Shaw cell [1-16]. These works have been extended to include effects arising from a constant rotation rate of the system and have mainly focused on the study of the linear growth rate, also, on the understanding fingering mechanisms of the interface when one of two immiscible fluids is accelerated toward the other under centrifugal forces. In this area, different configurations have been examined [2 - 5]. For instance, the linear stability analysis of an initially concentric circular drop in the case of high density and viscosity contrast was performed by Schwartz [2]. In his work, the coriolis force is included in an ad hoc manner and it was shown that circular drop is unstable both to a translation, and depending on the rotation rate, to a number of fingering modes. Later on, the linear stability analysis originally performed in [2] has been extended to an arbitrary density and viscosity contrast by carrillo et al., [3-5], and to a low viscosity contrast flows by Alvarez-Lacalle et al. [8]. In [3], Carrillo et al. have studied, both theoretically and experimentally, the behavior of the interface between two immiscible fluids in a rotating Hele-Shaw cell when the inner fluid is injected, with constant injection rate, through a hole at the rotation axis. Here, the linear growth rate calculated shows that the interfacial instability can be driven by the density difference between the fluids. In the case of vanishing injection rate and in the presence of centrifugal forcing, the nonlinear growth rate was also studied in [3] by authors. In the same spirit, Carrillo et al., [4,5], have investigated a series of experiments and have examined the radial displacement of a rotating fluid annulus in stable , [4], and unstable, [5], regimes. They have focused on the dynamics of axisymmetric annular configurations with air at the inner and outer layers and oil at the intermediate layer. By performing experiments in pre-wet and dry conditions, they have shown that the stability of the interfaces depends substantially on the wetting conditions at the leading interface. Later on, there has been interest of interfacial instabilities in ferroflu-

a e-mail: saniss@hotmail.com ids confined within Hele-Shaw cell [6-7]. Miranda [6] has treated the case in which the inner fluid is a ferrofluid in the presence of an external magnetic fields. Using a linear stability analysis and neglecting coriolis forces, he has determined the growth rate when both the centrifugal forces and magnetic forces are induced and has shown that the magnetic forces act to stabilize the fluid interface. Thereafter, Alvarez-Lacalle et al., [8], have studied the dynamics and morphology of the viscous fingering patterns formed at the circular interface between two immiscible fluids in a rotating Hele-Shaw cell in the low-viscosity contrast limit. These authors have found that, in addition to viscosity contrast and surface tension, the gap spacing of the cell also plays an important role in the linear regime to characterize finger competition processes. However, the influence of all these parameters (viscosity contrast, surface tension coefficient and dimensionless gap spacing) on the pattern morphology were addressed analytically by using a linear and weakly nonlinear stability analysis by Gadêlha and Miranda [9]. In this study the velocity gradients, which are related to internal friction in the fluid, are taken into account in the equilibrium condition. Following Schwartz [2], Waters and Cummings [10] have considered a flow in a rotating Hele-shaw cell taking into account the Coriolis forces. In the limit of high viscosity contrast and high density, They have determined the exact solution of the velocity field and the value of Eckman number corresponding to the fastest-growth rate. These results were compared with those obtained by Schwartz where the coriolis forces are included in a gap averaged two dimensional Darcy's law. Later, Abidate et al. [12] have reported analytical results for the development of instability of an interface between two immiscible, Newtonian fluid layers confined in a rotating annular Hele-Shaw cell. They have performed a linear stability analysis and focus their study on the influence of both Coriolis forces and curvature parameters on the interface instability growth rate. In this investigation it was shown that, the Coriolis force does not alter the stability of a disturbance with a particular wave number but reduces the maximum of the growth rate. The results related to the role played by the confinement of the liquid layers were also shown to provide a modification of the fastest-growing mode and its corresponding linear growth rate. 
All the studies cited above have examined the flow in an annular Hele-Shaw cell subject to a constant rotation. In this study, we focus attention on the interfacial instability in a Hele-Shaw cell under a time-dependent angular velocity. This study, is an extension of [12]. We examine the case of two immiscible, viscous and incompressible fluids in a time periodic rotating Hele-Shaw cell. In this situation, the basic flow is unsteady and the flow in each fluid is pulsed. Performing an inviscid linear stability analysis to describe the perturbation of the interface, we examine the effect of periodic rotation on the threshold of the interfacial instability.

\section{Formulation}

\subsection{Governing equations}

Consider two immiscible, incompressible Newtonian fluids confined in an annular Hele-Shaw cell of thickness $e$ and subject to a time-dependent angular velocity, $\boldsymbol{\Omega}(t)=$ $\Omega_{o}+\Omega_{m} \cos (\omega t) \mathbf{k}$, around its vertical symmetry axis. We denote by $\omega$ the dimensional frequency, $\Omega_{o}$ is a constant angular velocity, $\Omega_{m}$ is the amplitude of modulation and $\epsilon=\frac{e}{R} \ll 1$ the aspect ratio of the cell (see Fig. 1). Each

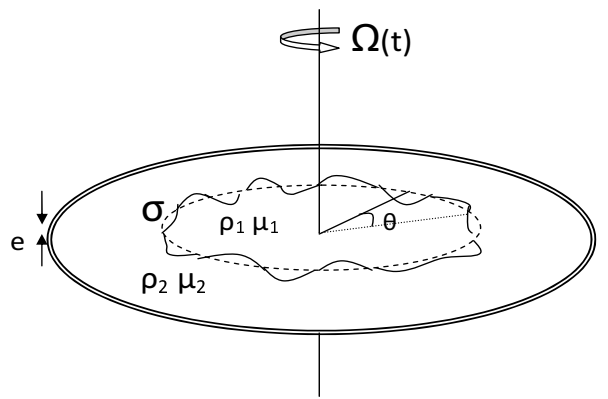

Fig. 1. Scheme arrangement of Hele-Shaw cell subject to a timedependent angular velocity.

fluid layer is characterized by the density $\rho_{j}$, the kinematic viscosity $v_{j}$, where the subscripts $j=1,2$ denotes the inner and outer layer respectively in the rotating frame.

The physical problem is governed by the following set of equations

$$
\begin{aligned}
& \nabla . \mathbf{V}_{j}=0 \quad(\mathrm{j}=1,2) \\
& \frac{d \mathbf{V}_{j}}{d t}=-\frac{1}{\rho_{j}} \nabla p_{j}+v_{j} \Delta \mathbf{V}_{j}-\mathbf{\Omega}(t) \wedge(\mathbf{\Omega}(t) \wedge \mathbf{r})-\frac{d \mathbf{\Omega}(t)}{d t} \wedge \mathbf{r}
\end{aligned}
$$

where $\mathbf{V}_{j}\left(u_{j}, v_{j}\right)$ is the fluid velocity, $p_{j}$ is the hydrodynamic pressure and $r$ the radial distance from the rotation axis.

\subsection{Base flow solution}

We assume that the equilibrium corresponds to a circular interface of radius $R$. Furthermore, the term $\frac{d \mathbf{\Omega}(t)}{d t} \wedge \mathbf{r}$ produces a basic velocity field having an azimuthal component $\left(0, V_{j}^{b}, 0\right)$. This basic solution satisfies necessarily the equation of continuity, the equations of momentum as well as the nonslip boundary conditions at the horizontal walls are,

$$
V_{j}^{b}=0 \text { at } z= \pm \frac{e}{2}
$$

Under these assumptions and as in the traditional HeleShaw flow, where the aspect ratio $\epsilon$ of the cell is considered smaller than unity, a first approximation of the base flow, $[17,18]$, is obtained from the system of equations (1)-(2) as follows

$$
\begin{aligned}
-\rho_{j} \frac{\left(V_{j}^{b}\right)^{2}}{r} & =-\frac{\partial P_{j}^{b}}{\partial r}+r \rho_{j} \Omega^{2}(t) \\
\rho_{j} \frac{\partial V_{j}^{b}}{\partial t} & =-\frac{1}{r} \frac{\partial P_{j}^{b}}{\partial \theta}+\mu_{j} \frac{\partial^{2} V_{j}^{b}}{\partial z^{2}}-\rho_{j} r \frac{d \Omega(t)}{d t} \\
0 & =-\frac{\partial P_{j}^{b}}{\partial z}
\end{aligned}
$$

\subsubsection{Base-state velocity}

Equations (4)-(6) are integrated to obtain the pressure and the velocity of the basic state. Therefore, the averaged components of velocity $\bar{V}_{j}^{b}$ can be written as

$$
\bar{V}_{j}^{b}=\bar{F}_{j} \cos (\omega t)+\bar{G}_{j} \sin (\omega t)
$$

the functions $\bar{F}_{j}$ and $\bar{G}_{j}$ are given by

$$
\begin{gathered}
\bar{F}_{j}=\frac{r \Omega_{m}}{\Gamma_{j}}\left[\frac{\sinh \left(\Gamma_{j}\right)+\sin \left(\Gamma_{j}\right)}{\cos \left(\Gamma_{j}\right)+\cosh \left(\Gamma_{j}\right)}-\Gamma_{j}\right] \\
\bar{G}_{j}=\frac{r \Omega_{m}}{\Gamma_{j}}\left[\frac{\sinh \left(\Gamma_{j}\right)-\sin \left(\Gamma_{j}\right)}{\cos \left(\Gamma_{j}\right)+\cosh \left(\Gamma_{j}\right)}\right]
\end{gathered}
$$

with $\Gamma_{j}=\left(\frac{\omega e^{2}}{2 v_{j}}\right)^{\frac{1}{2}}$ is the frequency number.

\subsubsection{Base-state pressure}

In this situation, the pressure $P_{j}^{b}$ of the base state is given by

$$
P_{j}^{b}=\frac{\rho_{j}}{2}\left[\left(\bar{V}_{j}^{b}\right)^{2}+r^{2} \Omega^{2}(t)\right]+C^{t e}
$$

where $C^{t e}$ is an arbitrary constant.

The pressure jump at the interface satisfies the LaplaceYoung equation given by

$$
P_{1}^{b}\left(R_{o}\right)-P_{2}^{b}\left(R_{o}\right)=\frac{\gamma}{R}
$$

Here, we denote by $\gamma$ the surface tension between the two fluids. 


\subsection{Linear stability}

We assume that the base state is disturbed so that the velocity and the pressure fields in the perturbed state are written as the sum of the base flow variables and small perturbations

$$
\mathbf{V}_{j}=\overline{\mathbf{V}}_{j}^{b}+\mathbf{v}_{j}(u(r, \theta, t), v(r, \theta, t)), P_{j}=P_{j}^{b}+p_{j}(r, \theta, t)
$$

The linear system of the conservation equations in the inviscid approximation is written as

$$
\begin{gathered}
\frac{\partial u_{j}}{\partial r}+\frac{u_{j}}{r}+\frac{1}{r} \frac{\partial v_{j}}{\partial \theta}=0 \\
\rho_{j}\left[\frac{\partial u_{j}}{\partial t}+\frac{V_{j}^{b}}{r} \frac{\partial u_{j}}{\partial \theta}-2 \frac{V_{j}^{b} v_{j}}{r}\right]=-\frac{\partial p_{j}}{\partial r} \\
\rho_{j}\left[\frac{\partial v_{j}}{\partial t}+\frac{V_{j}^{b}}{r} \frac{\partial v_{j}}{\partial \theta}+u_{j}\left(\frac{\partial V_{j}^{b}}{\partial r}+\frac{V_{j}^{b}}{r}\right)\right]=-\frac{1}{r} \frac{\partial p_{j}}{\partial \theta}
\end{gathered}
$$

following [12],we investigate the dynamical evolution of the interface in a rotating Hele-Shaw cell and we describe the instantaneous interface in polar coordinates as $R=R_{o}+$ $\xi(\theta, t)$, where $\xi(\theta, t)$ is an infinitesimal perturbation of the circular interface. Hereafter, to perform a stability analysis, we seek the solution of the linear system of Eqs. (12)-(14) in terms of normal modes

$$
\begin{aligned}
{\left[u_{j}, v_{j}, p_{j}\right] } & =\left[\tilde{u}_{j}, \tilde{v}_{j}, \tilde{p}_{j}\right] \exp (\operatorname{in} \theta) \\
\xi(\theta, t) & =\xi_{n}(t) \exp (\operatorname{in} \theta)
\end{aligned}
$$

with $i^{2}=-1, n$ is the azimuthal wave number.

We consider the velocity potentials $\Phi_{j}$, solutions of the continuity equation, Eq. (13), defined by

$$
\begin{gathered}
\phi_{1}=C_{1}(t) r^{n} \exp (\operatorname{in} \theta) \\
\phi_{2}=C_{2}(t) r^{-n} \exp (\operatorname{in} \theta)
\end{gathered}
$$

the constants $C_{j}(t)$ are determined using kinematics condition linearized at the interface,

$$
\frac{\partial \xi_{n}(t)}{\partial t}+i n \frac{\bar{V}_{j}^{b}}{r} \xi_{n}(t)=\tilde{u}_{j} \quad \text { at } \quad r=R
$$

To complete the set of equations we provide the dynamic boundary condition at the interface $r=R$

$$
\left(P_{1}^{b}+\tilde{p}_{1}\right)-\left(P_{2}^{b}+\tilde{p}_{2}\right)=\gamma \nabla \cdot \mathbf{n}
$$

Following $[6,12]$, we consider the curvature of the interface written in its linearized form as

$$
\nabla . \mathbf{n}=\frac{1}{R}\left[1-\left(1-n^{2}\right) \frac{\xi(\theta, t)}{R}\right]
$$

Hereafter, the total pressure is linearized at $r=R$ as follow

$$
\left(P_{j}^{b}+p_{j}\right)=p_{j}(R)+\left.\frac{\partial p_{j}}{\partial r}\right|_{r=R} \xi(\theta, t)+P_{j}^{b}(R)
$$

The linear stability problem, Eqns.(12)-(14), is reduced to an ordinary differential equation for the amplitude $\xi_{n}(t)$ of the interface displacement from its equilibrium position. Eq.(20) leads to a parametric differential equation

$$
\begin{aligned}
& \frac{d^{2} \xi_{n}(t)}{d t^{2}}+2 i\left[\alpha_{1}(n-1) \frac{\bar{V}_{1}^{b}}{R}+\alpha_{2}(n+1) \frac{\bar{V}_{2}^{b}}{R}\right] \frac{d \xi_{n}(t)}{d t} \\
+ & {\left[\frac{i n}{R}\left(\alpha_{1} \frac{d \bar{V}_{1}^{b}}{d t}+\alpha_{2} \frac{d \bar{V}_{2}^{b}}{d t}\right)-\alpha_{1} n(n-1)\left(\frac{\bar{V}_{1}^{b}}{R}\right)^{2}\right.} \\
- & \alpha_{2} n(n+1)\left(\frac{\bar{V}_{2}^{b}}{R}\right)^{2}+\left(\alpha_{2}-\alpha_{1}\right) n \Omega^{2}(t) \\
+ & \left.\frac{\gamma n\left(1-n^{2}\right)}{R^{3}\left(\rho_{2}+\rho_{1}\right)}\right] \xi_{n}(t)=0
\end{aligned}
$$

with $\alpha_{j}=\frac{\rho_{j}}{\rho_{1}+\rho_{2}}$. It is convenient to eliminate from (23) the term which contains the first order derivative in $\xi_{n}(t)$. To do so, we make the change of variable below

$$
\xi_{n}(t)=\bar{\xi}_{n}(t) \exp \left[-i \int\left(\alpha_{1}(n-1) \frac{\bar{V}_{1}^{b}}{R}+\alpha_{2}(n+1) \frac{\bar{V}_{2}^{b}}{R}\right) d t\right]
$$

Using Equation (24), the equation (23) is reduced to a nondimensional form by scaling space by $R$, time by $\sqrt{\frac{\gamma}{(\rho 1+\rho 2) R^{3}}}$, then, we obtain

$$
\begin{gathered}
\frac{d^{2} \bar{\xi}_{n}(t)}{d t^{* 2}}+\left[-n B_{o}+n\left(n^{2}-1\right)-2 n F r B_{o} \cos \left(\omega^{*} t^{*}\right)\right. \\
-\operatorname{Fr}^{2} B_{o}\left[\beta_{1} \cos ^{2}\left(\omega^{*} t^{*}\right)+\beta_{2} \sin ^{2}\left(\omega^{*} t^{*}\right)+\beta_{3} \sin \left(2 \omega^{*} t^{*}\right)\right] \\
\left.+i F r \omega^{*} \sqrt{-\frac{B_{o}}{A}}\left[\beta_{4} \cos \left(\omega^{*} t^{*}\right)+\beta_{5} \sin \left(\omega^{*} t^{*}\right)\right]\right] \bar{\xi}_{n}(t)=0
\end{gathered}
$$

whith

$$
\begin{aligned}
\beta_{1} & =n+\frac{F_{1}^{2}}{A}\left[\alpha_{1}^{2}(n-1)^{2}-n \alpha_{1}(n-1)\right]+\frac{F_{2}^{2}}{A}\left[\alpha_{2}^{2}(n+1)^{2}\right. \\
& \left.-n \alpha_{2}(n+1)\right]+2 \alpha_{1} \alpha_{2}\left(n^{2}-1\right) \frac{F_{1} F_{2}}{A} \\
\beta_{2} & =\frac{G_{1}^{2}}{A}\left[\alpha_{1}^{2}(n-1)^{2}-n \alpha_{1}(n-1)\right]+\frac{G_{2}^{2}}{A}\left[\alpha_{2}^{2}(n+1)^{2}\right. \\
& \left.-n \alpha_{2}(n+1)\right]+2 \alpha_{1} \alpha_{2}\left(n^{2}-1\right) \frac{G_{1} G_{2}}{A} \\
\beta_{3} & =\frac{F_{1} G_{1}}{A}\left[\alpha_{1}^{2}(n-1)^{2}-n \alpha_{1}(n-1)\right]+\frac{F_{2} G_{2}}{A}\left[\alpha_{2}^{2}(n+1)^{2}\right. \\
& \left.-n \alpha_{2}(n+1)\right]+\frac{\alpha_{1} \alpha_{2}}{A}\left(n^{2}-1\right)\left(F_{1} G_{2}+F_{2} G_{1}\right) \\
\beta_{4} & =\left(\alpha_{1} G_{1}-\alpha_{2} G_{2}\right) \\
\beta_{5} & =\left(\alpha_{2} F_{2}-\alpha_{1} F_{1}\right)
\end{aligned}
$$

where $B_{o}=\frac{\left(\rho_{1}-\rho_{2}\right) R^{3} \Omega_{o}^{2}}{\gamma}$ is the Bond number, $F_{r}=\frac{\Omega_{m}}{\Omega_{o}}$ the Froude number, $A=\frac{\rho_{2}-\rho_{1}}{\rho_{2}+\rho_{1}}$ the Atwood number. The parameters $\alpha_{1}$ and $\alpha_{2}$ can be expressed as a function of the Atwood number with $\alpha_{1}=\frac{1+A}{2}$ and $\alpha_{2}=\frac{1-A}{2}$.

Remark that in the limit case corresponding to $F r=0$, the solution determined in this work, and given by equations (25), tends to the solution corresponding to the marginal stability equation of Miranda [6] (equation (2) in [6] without magnetic bond number $N_{B}$ ). 


$$
B_{o}-\left(n^{2}-1\right)=0
$$

The equation (25) is solved numerically using the spectral method in MATLAB, this method provides a matrix formulation of the linear stability problem which corresponds to a nonlinear eigenvalue problem.

\section{Results and Discussion}

In this section, we present the results corresponding to the case $\frac{\Omega_{m}}{\Omega_{o}} \ll 1(F r \ll 1)$. In this situation the equation (25) can be approximated by:

$$
\frac{d^{2} \xi_{n}}{d \tau^{2}}+\frac{4}{\omega^{*}}\left[-n B_{o}+n\left(n^{2}-1\right)-2 n F r B_{o} \cos (2 \tau)\right] \xi_{n}=0
$$

This equation corresponds to a classical Mathieu equation which is solved numerically to determine the marginal stability curves $B_{o}(n)$. Notice that, the interface can be linearly unstable to a Rayleigh-Taylor instability and successive parametric instability regions. In this situation, corresponding to small Froude number and small dimensionless frequency, the parametric instability regions vanish and only the Rayleigh-Taylor instability occurs.

In figure (2), we illustrate the marginal stability curves for

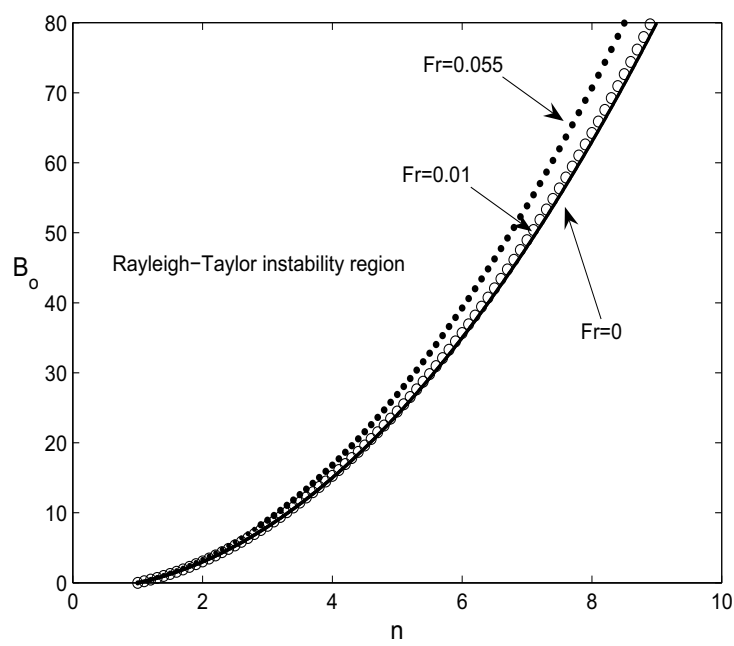

Fig. 2. Variation of the Bond number, $B_{o}$, versus the wave number, $n$, for $\omega^{*}=0.1$ and different values of the Froude number $F_{r}$

assigned value of the dimensionless frequency, $\omega^{*}=0.1$, and for different Froude number $F_{r}$. It turns out that an increase in the Froude number, corresponds to increasing the amplitude modulation of the angular velocity $\Omega_{m}$, acts to stabilize the interface. Furthermore, it is worth noting that, when the Froude number decreases the marginal stability curve converge toward that obtained by Miranda [6] corresponding to $F_{r}=0$.

\section{Conclusion}

In this study, we have performed a linear stability analysis of an interface between two viscous immiscible fluids of different densities confined in a time-periodic rotating Hele-Shaw cell. The linear problem is reduced to a periodic Mathieu equation governing the evolution of the amplitude of the interface. In this investigation, we have studied the limiting case $F_{r} \ll 1$, corresponding to small modulation amplitude of the time-periodic rotation $\frac{\Omega_{m}}{\Omega_{0}} \ll 1$. We have focused our analysis on the effect of the Froude number, $F_{r}$, on the boundaries of the marginal stability and it was shown that, an increase in this number has a stabilizing effect on the Rayleigh-Taylor instability region.

\section{References}

[1] P. G. Saffman and G. I. Taylor, Proc. R. Soc. London, Ser. A 245, 312 (1958).

[2] L. W. Schwartz, Phys. Fluids. 1, 167 (1989).

[3] L. Carrillo, F. X. Magdaleno, J. Casademunt, and J. Ortin, Phys.Rev. E 54, 6260 (1996).

[4] L. Carrillo, J. Soriano, and J. Ortin, Phys. Fluids. 11, 778 (1999).

[5] L. Carrillo, J. Soriano, and J. Ortin, Phys. Fluids. 12, 1685 (2000).

[6] J. A. Miranda, Phys. Rev. E62, 2985 (2000).

1. Jackson D. J. Jackson, J. A. Miranda, Phys. Rev. E 67,017301 pp. 1-4,(2003).

[8] E. Alvarez-Lacalle, J. Ortin and J. Casademunt, Phys. Fluids. 16, 908 (2004).

[9] H. Gadêlha and J. A. Miranda, Phys. Rev. E 70, 066308 (2004).

[10] S. L. Waters and L. J. Cummings, Phys. Fluids 17, 048101 (2005).

[11] S. L. Waters, L. J. Cummings, K. M. Shakesheff, F. R. A. J. Rose, IMA J. Math. Appl. Med. Biol. 23, 311 (2006).

[12] A. Abidate, S. Aniss, O. Caballina, M. Souhar, Phys. Rev. E, 75, 046307 (2007).

[13] H. Gadêlha, N. Brito, J. A. Miranda, Phys. Rev. E75, 016305 pp. 1-9, (2007).

[14] E. Alvarez-Lacalle, H. Gadêlha, J. A. Miranda, Phys. Rev. E78, 026305 pp. 1-10, (2008).

[15] J. A. Miranda, H. Gadêlha, A. T. Dorsey, Phys. Rev. E 82, 066306 pp. 1-10, (2010).

[16] E. O. Dias, J. A. Miranda, Phys. Rev. E 87, 053014 pp. 1-7, (2013).

[17] S. Aniss, M. Souhar, J. P. Brancher, Phys. Fluids 7, pp. 926-934 (1995).

[18] S. Aniss, M. Souhar, M. Belhaq, Phys. Fluids 12(2), pp. 262-268 (2000). 